\title{
Performance of Homologous and Heterologous Prime-Boost Immunization Regimens of Recombinant Adenovirus and Modified Vaccinia Virus Ankara Expressing an Ag85B-TB10.4 Fusion Protein against Mycobacterium tuberculosis ${ }^{\mathbb{S}}$
}

\author{
Yiming Kou ${ }^{1}$, Mingming $\mathrm{Wan}^{1}$, Wei Shi ${ }^{1}$, Jie Liu ${ }^{1}$, Zhilei Zhao ${ }^{1}$, Yongqing $\mathrm{Xu}^{1}$, Wei Wei ${ }^{1}$, Bo Sun ${ }^{1,2}$, Feng Gao ${ }^{1,2}$, \\ Linjun Cai $^{1,2 *}$, and Chunlai Jiang ${ }^{1,2 *}$ \\ ${ }^{1}$ National Engineering Laboratory for AIDS Vaccine, School of Life Science, Jilin University, Changchun 130012, P.R. China \\ ${ }^{2}$ Key Laboratory for Molecular Enzymology and Engineering of the Ministry of Education, School of Life Science, Jilin University, Changchun \\ 130012, P.R. China
}

Received: December 28, 2017

Revised: April 1, 2018

Accepted: April 3, 2018

April 27, 2018

${ }^{*}$ Corresponding authors

L.C.

Phone: +86-431-85167790;

Fax: +86-431-85167751;

C.J.

Phone: +86-431-85167790;

Fax: +86-431-85167751;

E-mail: jiangcl@jlu.edu.cn

S upplementary data for this paper are available on-line only at http://jmb.or.kr.

pISSN 1017-7825, eISSN 1738-8872

Copyright@ 2018 by

The Korean Society for Microbiology and Biotechnology
First published online

E-mail: linjun_cai@jlu.edu.cn

Tuberculosis (TB) remains a serious health issue around the word. Adenovirus (Ad)-based vaccine and modified vaccinia virus Ankara (MVA)-based vaccine have emerged as two of the most promising immunization candidates over the past few years. However, the performance of the homologous and heterologous prime-boost immunization regimens of these two viral vector-based vaccines remains unclear. In the present study, we constructed recombinant Ad and MVA expressing an Ag85B-TB10.4 fusion protein (AdH4 and MVAH4) and evaluated the impact of their different immunization regimens on the humoral and cellular immune responses. We found that the viral vector-based vaccines could generate significantly higher levels of antigen-specific antibodies, IFN- $\gamma$-producing splenocytes, $\mathrm{CD} 69^{+} \mathrm{CD} 8^{+} \mathrm{T}$ cells, and IFN- $\gamma$ secretion when compared with bacillus Calmette-Guérin (BCG) in a mouse model. AdH4-containing immunization regimens (AdH4-AdH4, AdH4-MVAH4, and MVAH4-AdH4) induced significantly stronger antibody responses, much more IFN- $\gamma$-producing splenocytes and $\mathrm{CD} 9^{+} \mathrm{CD} 8^{+} \mathrm{T}$ cells, and higher levels of IFN- $\gamma$ secretion when compared with the MVAH4-MVAH4 immunization regimen. The number of IFN- $\gamma$-producing splenocytes sensitive to CD8 ${ }^{+}$T-cell restricted peptides of Ag85B (9-1p and 9-2p) and Th1-related cytokines (IFN- $\gamma$ and TNF- $\alpha$ ) in the AdH4-MVAH4 heterologous prime-boost regimen immunization group was significantly higher than that in the other viral vector-based vaccine- and BCGimmunized groups, respectively. These results indicate that an immunization regimen involving AdH4 may have a higher capacity to induce humoral and cellular immune responses against TB in mice than that by regimens containing BCG or MVAH4 alone, and the AdH4-MVAH4 prime-boost regimen may generate an ideal protective effect.

Keywords: Mycobacterium tuberculosis, adenovirus-based vaccine, vaccinia virus, bacillus Calmette-Guérin, Ag85B, TB10.4

\section{Introduction}

Tuberculosis (TB) remains a serious global health issue. It caused illness in 10.4 million people and accounted for 1.3 million deaths in 2016 [1]. Bacillus Calmette-Guérin (BCG), the only anti-TB vaccine currently licensed, has been used worldwide since 1921, but its efficacy varies wildly among different populations. The efficacy of BCG for fighting adult pulmonary TB is reportedly limited, and the vaccine may potentially interfere with the tuberculin skin test 
reactivity [2]. The situation has been further complicated with the increased prevalence of drug-resistant and multidrug-resistant TB [3]. Therefore, a more effective vaccine against $\mathrm{TB}$ is urgently needed that would prevent drug-resistant TB in all age groups [4-6].

Currently, a few new types of vaccines to prevent TB disease are under development, including subunit vaccines, DNA vaccines, and viral vector-based vaccines. A robust cellular immunity is necessary for Mycobacterium tuberculosis (M.tb) since it is an intracellular pathogen. Therefore, enhancing the cellular immunity is a strategy for more effective vaccine development against M.tb [7]. HyVac4 and Hybrid56 are two vaccines in phase II clinical trials. Both of them are recombinant fusion proteins and use the adjuvant IC31, which can induce potent cellular immune responses via Toll-like receptor 9. Besides the cellular immunity induced by a particular adjuvant, viral-based vaccines also can induce cellular immune responses inherently. The recombinant adenovirus (Ad) vaccine and genetically modified vaccinia virus Ankara (MVA) vaccine are the two major forms of viral vector-based vaccines, and several variations of these vaccines are currently making their way to clinical trials [8-11].

The Ag85 complex, ESAT6, and TB10.4 are major antigens of M.tb and have been ascertained as the most potent antigen species for TB vaccine design [12]. All of them have been chosen as antigens for vaccine design [13-15]. The Ag85 complex containing Ag85B, Ag85A, and Ag85C is the most abundant set of antigens in the culture supernatant of mycobacteria, accounting for almost half of the total amount of extracellular proteins, with the Ag85B protein corresponding to $22 \%$, the Ag85A protein corresponding to $15 \%$, and the Ag85C protein corresponding to $8 \%$ [16]. Therefore, $\mathrm{Ag} 85 \mathrm{~B}$ is the primary extracellular protein of M.tb. It is likely that Ag85B is more important than Ag85A and Ag85C because of its abundant secretion compared with the other members of this complex and its presence in the culture after 3 days [17]. Several studies have shown that the Ag85B-ESAT6 fusion protein-based vaccine is an effective vaccine for tuberculosis prevention [18-20]. However, because ESAT6 is a valuable reagent for TB diagnostics, identifying a novel vaccine that does not involve the ESAT6 protein is a priority [21].

The vaccination regimen is an important factor that can influence the efficacy of a vaccine. In our previous study, we evaluated heterologous prime-boost regimens by priming with BCG or recombinant BCG and boosting with recombinant MVA and/or Ad. We found that boosting mice with Ad85B-ESAT6 and MVA85B-ESAT6 vaccines could strengthen the BCG-induced immune protective effect [7]. These data imply that the immunization regimen has some impact on TB vaccine efficacy. However, our study described results in BCG-primed mice. Without BCG priming, the performance of different prime-boost immune regimens of Ad and MVA expressing the Ag85B-TB10.4 vaccines against $M$.tb have not been evaluated yet.

Therefore, in the present study, we selected Ag85B and TB10.4 as vaccine antigens because these proteins are promising vaccine candidates against $\mathrm{TB}$ and a vaccine HyVac4 based on a TB10.4 and Ag85B fusion protein is currently in clinical trials [22-24]. Next, we constructed two viral vector-based vaccines (recombinant Ad and MVA expressing the Ag85B-TB10.4 fusion protein, named AdH4 and MVAH4, respectively) and evaluated the performance of the various prime-boost immune regimens of these two vaccines.

\section{Materials and Methods}

\section{Construction of MVAH4 and AdH4}

The recombinant MVA expressing the Ag85B-TB10.4 fusion protein (MVAH4) was constructed and purified as described previously [25]. The recombinant replication-defective Ad expressing Ag85B-TB10.4 (AdH4) was constructed according our previous work by using the AdMax Adenovirus Creation Kit (MicrobixBiosystems, Canada) [26]. Briefly, the Ag85B-TB10.4 fusion gene was cloned into pDC316. Then, pDC316-Ag85B-TB10.4 was co-transfected with genomic plasmid (pBHGlox $\Delta \mathrm{E} 1,3 \mathrm{Cre}$ ) into HEK-293 cells and the AdH4 was rescued by homologous recombination. The virus was stored at $-80^{\circ} \mathrm{C}$ and the titers were determined as $\mathrm{TCID}_{50}$ on HEK-293 cells before immunization.

\section{Antigen Proteins and Peptides}

The recombinant Ag85B and TB10.4 proteins were produced by Escherichia coli. The antigen peptides of Ag85B (9-1p, 9-2p, 18-1p, and 18-2p) and TB10.4 (P2, P3, P7, and P8) were synthesized by GL Biochem (China) [26, 27].

\section{Immunization}

Female BALB/c mice were procured from Changsheng Biotechnology Co. LTD (China), randomly assigned into six groups (5 mice per group), and kept in individual ventilated cages at the Experimental Animal Center Laboratory of Jilin University. Mice were subcutaneously (s.c.) injected once at the base of the thighs with BCG $\left(1 \times 10^{6} \mathrm{CFU}\right)$ or twice with viral vector vaccines $\left(1 \times 10^{7} \mathrm{pfu} \mathrm{MVAH} 4\right.$ s.c. or $1 \times 10^{7} \mathrm{pfu}$ AdH4 intramuscularly) at 2-week intervals. The vaccine regimens are described in detail in Fig. 1. All the animal experiments were approved by the Institutional Animal Care and Use Committee of Jilin University. 


$\begin{array}{llll}\text { Groups } & \text { Day0 } & \text { Day14 } & \stackrel{\text { Day28 }}{\longrightarrow} \\ \text { BCG } & \stackrel{1}{ } & \\ \text { MVAH4-MVAH4 } & \text { BCG(s.c.) } & & \\ \text { AdH4-AdH4 } & \text { AdH4(i.m.) } & \text { AdH4(i.m.) } & \text { sacrifice } \\ \text { MVAH4-AdH4 } & \text { MVAH4(s.c.) } & \text { AdH4(i.m.) } & \\ \text { AdH4-MVAH4 } & \text { AdH4(i.m.) } & \text { MVAH4(s.c.) } & \end{array}$

Fig. 1. Schematic diagram of the experimental design.

Mice (5 mice per group) were vaccinated with different immunization regimens. The BCG group was injected once subcutaneously (s.c.) with BCG $\left(1 \times 10^{6} \mathrm{CFU}\right)$ at the base of the thighs on day 0 . The viral vector-based vaccines were injected on day 0 and day 14 with $1 \times 10^{7} \mathrm{pfu} /$ dose. MVAH4 and AdH4 were given s.c. and intramuscularly (i.m.), respectively. Then, the mice were sacrificed on day 28. BCG: bacillus Calmette-Guérin; AdH4: recombinant adenovirus expressing the Ag85B-TB10.4 fusion protein; MVAH4: recombinant modified vaccinia virus Ankara expressing the Ag85B-TB10.4 fusion protein.

\section{Enzyme-Linked Immunosorbent Assay (ELISA)}

ELISA was carried out for the measurement of antigen-specific antibodies. The 96-well plates were coated with $5 \mu \mathrm{g} / \mathrm{ml}$ (100 $\mu \mathrm{l} /$ well) recombinant Ag85B or TB10.4 protein and incubated overnight. The 50 -fold diluted sera in PBS containing $1 \%$ bovine serum albumin was added to each antigen-coated well, and then 1:1,000 diluted primary antibodies (goat anti-mouse IgG, IgG2a,or IgG1; Sigma, Germany) were applied. Thereafter, 1:2,000 diluted secondary antibodies (HRP-conjugated rabbit anti-goat IgG) were applied and revealed using TMB substrate for $15 \mathrm{~min}$ and stopped with $2 \mathrm{M} \mathrm{H}_{2} \mathrm{SO}_{4}$. The optical density (OD) was measured with a microplate reader (iMark; Bio-Rad, USA) at $450 \mathrm{~nm}$.

\section{Isolation of Splenocytes}

Splenocytes were isolated according to previous literatures [25]. Briefly, mouse spleens were separated aseptically and grinded through a $75-\mu \mathrm{m}$ mesh nylon sieve into RPMI-1640 medium containing $10 \%$ fetal bovine serum (FBS). The erythrocytes were removed by adding ammonium-chloride-potassium buffer and the splenocytes were resuspended in the culture medium $\left(1 \times 10^{7}\right.$ cells $\left./ \mathrm{ml}\right)$.

\section{IFN- $\gamma$ ELISpot Assay}

IFN- $\gamma$ ELISpot assays were carried out according to the manufacturer's protocol (BD Biosciences, USA). Briefly, 96-well plates were coated with anti-mouse IFN- $\gamma \mathrm{mAb}$ and blocked with RPMI-1640 containing 10\% FBS. Thereafter, the splenocytes were added $\left(1 \times 10^{6}\right.$ cells /well $)$ and stimulated with different peptides (9-1p, 9-2p, 18-1p, 18-2p, P2, P3, P7, and P8; final concentration: $5 \mu \mathrm{g} / \mathrm{ml}$ ). After $24 \mathrm{~h}$ incubation at $37^{\circ} \mathrm{C}$, the cells were removed by washing. Next, the plates were incubated with biotinylated antimouse IFN- $\gamma \mathrm{mAb}$, followed by incubation with streptavidinHRP. The spots were developed by the AEC substrate.

\section{Flow Cytometric Analyses}

Cell surface markers of splenocytes were stained as described previously. [25] The splenocytes were stained with CD4-APC, CD8-PerCP, CD3-FITC, and CD69-PE (BioLegend, USA). Briefly, $1 \times 10^{6}$ cells were stimulated for $24 \mathrm{~h}$ with $5 \mu \mathrm{g} / \mathrm{ml}$ antigen. $\mathrm{Fc}$ receptors were blocked with $1 \mu \mathrm{g}$ of anti-mouse CD16/CD32, and then the cells were stained with anti-CD3-FITC, CD4-APC, CD8PerCP, and CD69-PE antibodies for surface markers. Finally, the cells were washed with cell staining buffer, and analyzed by BD Accuri C6 flow cytometry. The results were analyzed using FlowJo software.

\section{Analysis of Cytokine Levels}

Splenocytes were cultured in 24-well plates $\left(5 \times 10^{6}\right.$ cells/well) with stimulation of the Ag85B or TB10.4 protein $(5 \mu \mathrm{g} / \mathrm{ml})$ for $48 \mathrm{~h}$. For detection of cytokines in the culture supernatants, a mouse Th1/Th2 Panel Multi-analyte Flow Assay Kit (BioLegend, USA) was used according to the manufacturer's protocol. IFN- $\gamma$, TNF- $\alpha$, IL-4, IL-5, IL-6, and IL-10 levels were analyzed using LEGENDplex ver. 7.0 (VigeneTech, USA) software.

\section{Statistical Analyses}

Data are presented as the mean $\pm S D$, and the Student's $t$-test was performed for independent samples. For the above statistical analysis, GraphPad Prism 7 was used. $P$ values less than 0.05 were considered significant.

\section{Results}

\section{Antigen-Specific Antibody Responses Elicited by Different Vaccine Regimens}

In order to evaluate the humoral immune response induced by the vaccines, antigen-specific antibody levels in mouse sera were measured by ELISA. As shown in Fig. 2, the levels of anti-Ag85B and anti-TB10.4 IgG, IgG2a, and IgG1 in all mice immunized with the viral vector-based vaccines were significantly higher than those in BCG-immunized mice. Furthermore, the heterologous prime-boost immunization regimens (AdH4-MVAH4 or MVAH4-AdH4) and the AdH4-AdH4 homologous prime-boost regimen induced significantly stronger antigen-specific antibody responses than that by homologous MVAH4-MVAH4 vaccination. No significant change in the $\operatorname{IgG} 2 \mathrm{a} / \mathrm{IgG} 1$ ratio was observed among all the groups. These data indicate that both viral vector-based vaccines can induce mouse humoral immune response against Ag85B-TB10.4, and all these vaccines elicited a balanced Th1 and Th2 immune response.

\section{Antigen-Specific IFN- $\boldsymbol{\gamma}$-Producing Splenocytes}

Splenocytes were cocultured with antigenic peptides (9- 
A
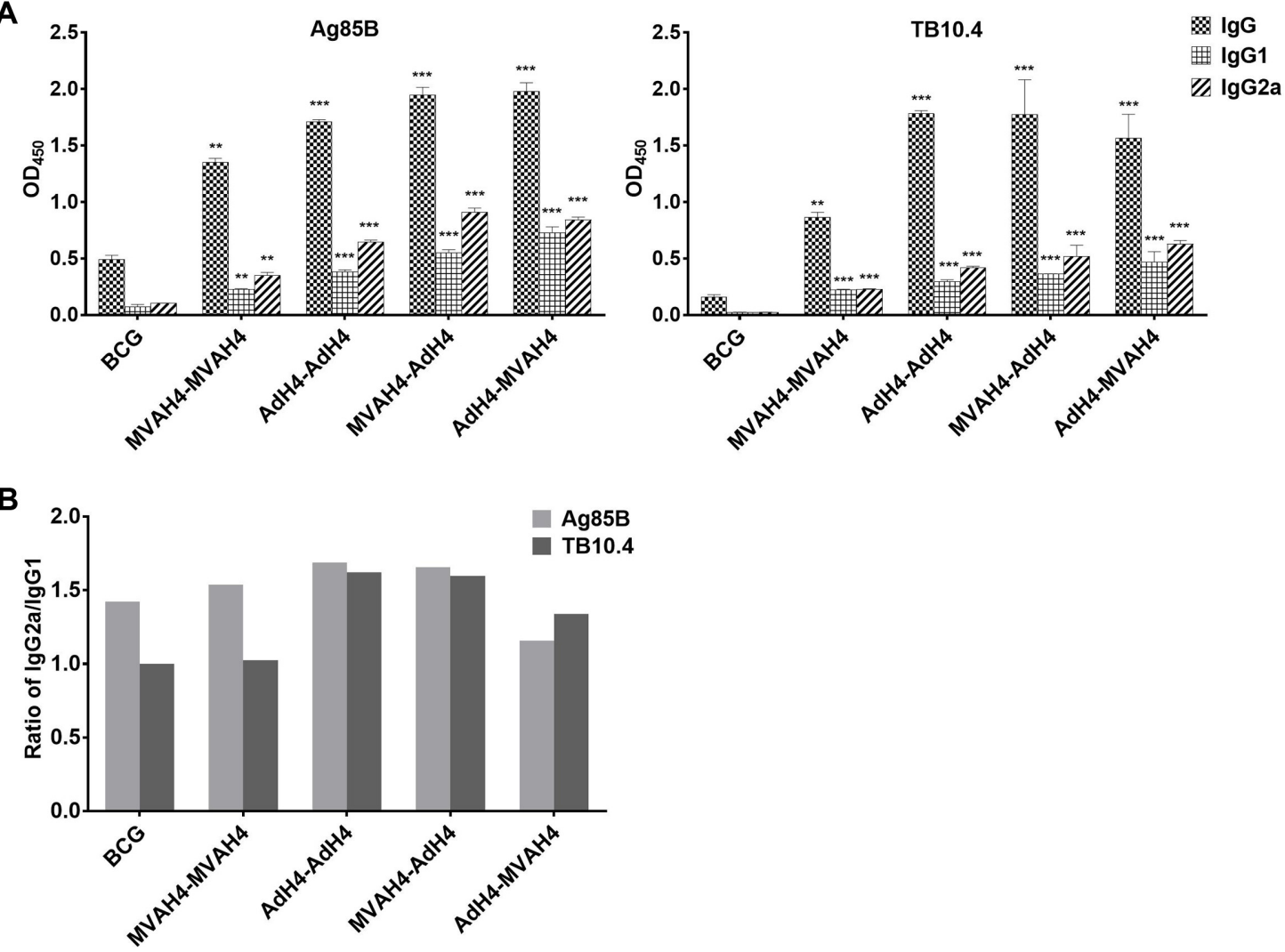

Fig. 2. Levels of antigen-specific antibodies (total IgG, IgG1, and IgG2a) and the ratio of IgG2a/IgG1.

(A) $\mathrm{OD}_{450}$ values of antigen-specific total IgG, IgG1, and IgG2a. Serum samples were collected on day 28 from immunized mice and the levels of Ag85B- or TB10.4-specific IgG, IgG1, and IgG2a were measured by ELISA. The serum samples were diluted 50-fold with assay buffer. ${ }^{* *} p<0.01$; $* * * p<0.001$. (B) Ratio of IgG2a/IgG1.

1p, 9-2p, 18-1p, 18-2p, P2, P3, P7, and P8), and then the IFN- $\gamma$-producing cells were measured by the ELISpot assay. The viral vector-based vaccine induced much more antigen-specific IFN- $\gamma$-producing splenocytes than that induced by BCG (Fig. 3). We also found that much more IFN- $\gamma$-producing splenocytes were activated by $18-1 \mathrm{p}$ and 18-2p (CD4 T-cell restricted peptides of Ag85B) and P2 and P3 (CD8 T-cell restricted peptides of TB10.4). The number of IFN- $\gamma$-producing splenocytes that were sensitive to Ag85B peptides (18-1p, 18-2p, 9-1p, and 9-2p) and TB10.4 peptides (P2 and P3) in all viral vector-based vaccine vaccination groups was significantly higher than that in the BCG-immunized group. The number of IFN- $\gamma$-producing splenocytes that were sensitive to TB10.4 peptides (P7 and P8) in AdH4-containing vaccine immunization groups was significantly higher than that in the MVAH4-MVAH4immunized mice and BCG-immunized mice. We also found that much more P8 peptide-sensitive IFN- $\gamma$-producing splenocytes were produced than other peptide-sensitive
IFN- $\gamma$-producing splenocytes in the BCG-immunized mice, which is similar to previous studies [27]. Furthermore, the

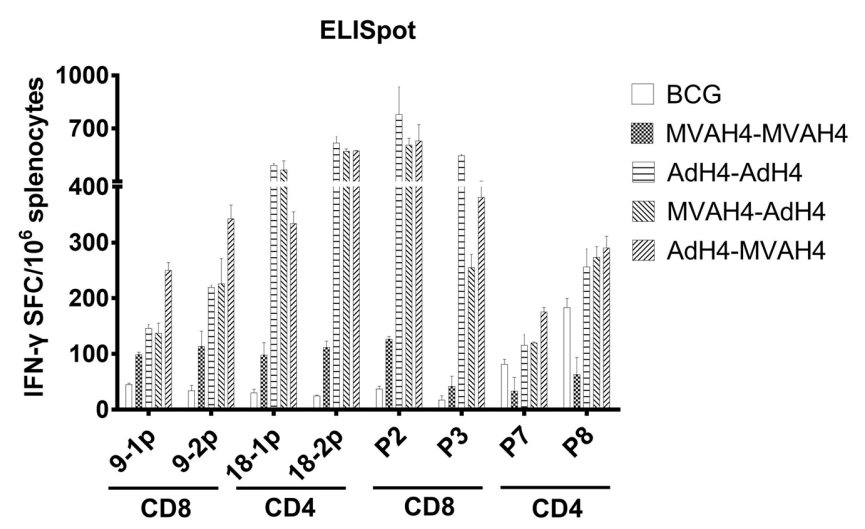

Fig. 3. Results of the IFN- $\gamma$ ELISpot assay.

The frequencies of antigen-specific IFN- $\gamma$-secreting cells were assayed ex vivo via the IFN- $\gamma$ ELISpot assay using freshly isolated splenocytes on day 28. Data shown are the mean \pm standard deviation for two independent assays. 
A

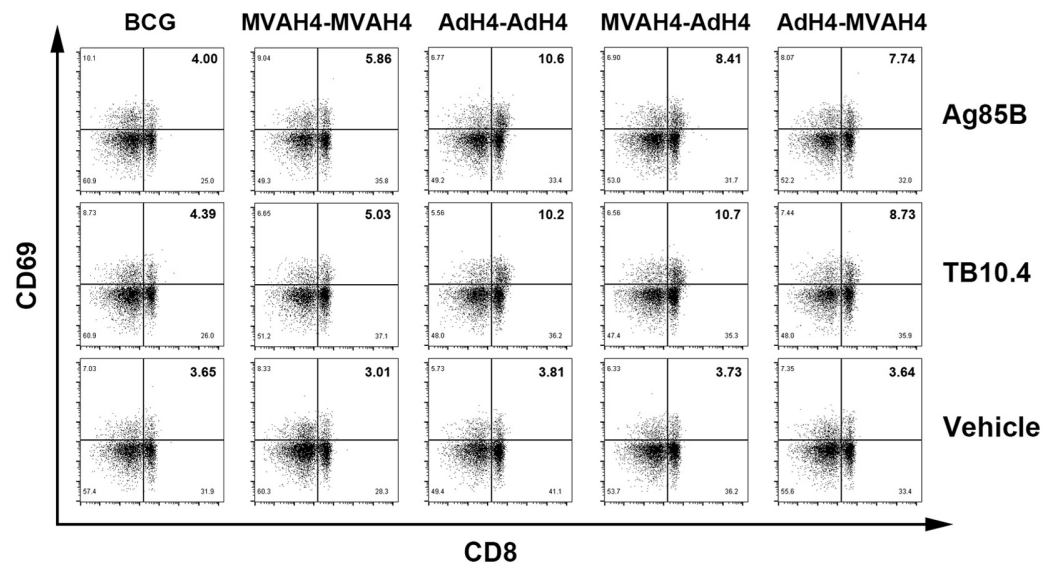

B

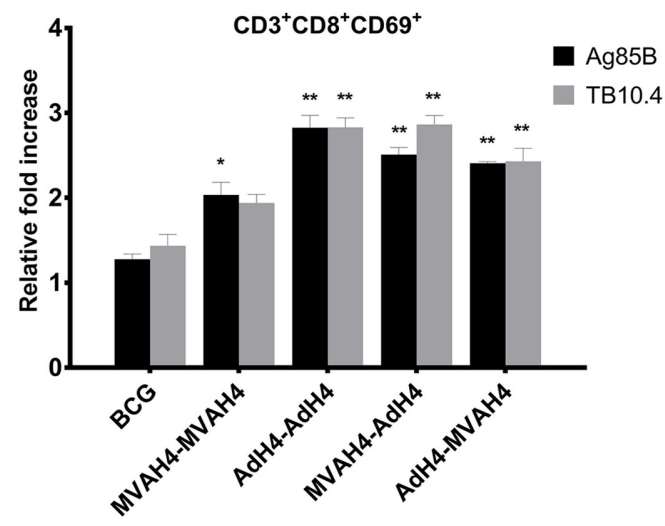

Fig. 4. CD69 expression on the surface of T cells.

(A) Representative flow cytometry analysis results of CD69 expression on T cells. Splenocytes were stimulated without or with a specific antigen $\left(5 \mu \mathrm{g} / \mathrm{ml} \mathrm{Ag} 85 \mathrm{~B}\right.$ or TB10.4 protein) overnight in vitro, and measured by flow cytometry. The values represent the percentages of CD69 $9^{+} \mathrm{CD} 8^{+}$cells in T cells. (B) The relative fold increase of $\mathrm{CD} 69^{+} \mathrm{CD} 8^{+}$cells is the ratio of the percentage of $\mathrm{CD} 69^{+} \mathrm{T}$ cells in antigen (Ag85B or TB10.4 protein)treated $\mathrm{T}$ cells to the percentage of $\mathrm{CD} 9^{+} \mathrm{T}$ cells in control solvent-treated $\mathrm{T}$ cells. Data are representative of two independent experiments.

number of IFN- $\gamma$-producing splenocytes that were sensitive to CD8 T-cell restricted peptides of Ag85B (9-1p and 9-2p) in the AdH4-MVAH4 heterologous prime-boost regimenimmunized group was significantly higher than that in the other viral vector-based vaccine-immunized groups. These data imply that the Ad-based vaccines might have a higher capacity to induce mice cellular immune responses against TB than BCG and MVAH4 vaccines.

\section{CD69 Surface Expression on T cells}

In order to characterize the phenotype of antigen-specific activated $\mathrm{T}$ cells, splenocytes were stimulated with or without the antigens in vitro, and the expression of CD3, CD4, CD8, and CD69 was analyzed by flow cytometry analysis. As shown in Fig. 4, compared with the BCG immunized group, Ag85B stimulation could significantly increase the populations of $\mathrm{CD} 69^{+} \mathrm{CD} 8^{+} \mathrm{T}$ cells in the viral vector-based vaccine-immunized groups. The TB10.4 stimulation could significantly increase the populations of $\mathrm{CD} 9^{+} \mathrm{CD}^{+} \mathrm{T}$ cells in the AdH4-AdH4-, MVAH4-AdH4-, and AdH4-MVAH4-immunized groups when comparing with the BCG-immunized group. Both Ag85B and TB10.4 stimulations could slightly increase the populations of $\mathrm{CD} 9^{+} \mathrm{CD}^{+} \mathrm{T}$ cells in the AdH4-AdH4-, MVAH4-AdH4-, and AdH4-MVAH4-immunized groups than that in the MVAH4-MVAH4-immunized group, but not significantly. After Ag85B and TB10.4 stimulations, the proportions of $\mathrm{CD} 69^{+} \mathrm{CD}^{+} \mathrm{T}$ cells in all immunized groups were not significantly different from each other (Fig. S1). All these data indicate that the viral vector-based vaccines might have more potential to activate $\mathrm{CD} 8^{+} \mathrm{T}$ cells than that by BCG, and the Ad-containing immunization regimens could activate $\mathrm{CD} 8^{+} \mathrm{T}$ cells more easily than that by the MVAH4MVAH4 vaccine regimen.

\section{Th1/Th2 Cytokine Profiles}

Splenocytes were stimulated with Ag85B and TB10.4 $(5 \mu \mathrm{g} / \mathrm{ml})$ for $48 \mathrm{~h}$, and then cytokines in the culture supernatants were measured using mouse Th1/Th2 Panel Multi-analyte Flow Assay kits. Significant differences in the levels of Th1/Th2 cytokines were observed among these prime-boost immunization regimens (Table 1). We found that the levels of Th1-related cytokines (IFN- $\gamma$ and TNF- $\alpha$ ) in the AdH4-MVAH4 heterologous prime-boost regimen-immunized group were significantly higher than those in the BCG-immunized group. We also found that Ag85B stimulation can induce Th1 cytokine (IFN- $\gamma$ and TNF- $\alpha$ ) secretion at higher levels in the viral vector-based vaccine-immunized mice than in BCG-immunized mice. Additionally, the levels of IFN- $\gamma$ in mice that had been administered vaccines containing AdH4 were significantly higher than that in MVAH4-MVAH4-immunized mice. However, the highest level of TNF- $\alpha$ was observed in MVAH4-MVAH4-immunized mice. Similar secretion patterns of IFN- $\gamma$ and TNF- $\alpha$ were observed when mice were stimulated with TB10.4. Table 1 also shows that Ag85B 
Table 1. Th1/Th2 cytokine profiles.

\begin{tabular}{|c|c|c|c|c|c|c|}
\hline Stimulation & Cytokines & BCG & MVAH4-MVAH4 & AdH4-AdH4 & MVAH4-AdH4 & AdH4-MVAH4 \\
\hline \multirow[t]{6}{*}{ Ag85B } & IFN- $\gamma$ & $734 \pm 35$ & $1032 \pm 8^{*}$ & $15,480 \pm 538^{*}$ & $11,971 \pm 56^{*}$ & $6,168 \pm 361^{*}$ \\
\hline & TNF- $\alpha$ & $1.75 \pm 0.37$ & $19.02 \pm 0.13^{*}$ & $2.41 \pm 0.13$ & $2.17 \pm 0.13$ & $17.08 \pm 2.29^{*}$ \\
\hline & IL-4 & $36 \pm 2.0$ & $3 \pm 0.2$ & $78 \pm 4.7^{*}$ & $70 \pm 3.6^{*}$ & $43 \pm 8.1$ \\
\hline & IL-5 & $322 \pm 33.9$ & $126.5 \pm 4.5$ & $5,508 \pm 197^{*}$ & $4,283 \pm 383^{*}$ & $1,427 \pm 55^{*}$ \\
\hline & IL-6 & $37 \pm 2.3$ & $207 \pm 4.1^{*}$ & $196 \pm 12.1^{*}$ & $167 \pm 6.9^{*}$ & $239 \pm 14^{*}$ \\
\hline & IL-10 & $39 \pm 3.1$ & $57 \pm 3.2$ & $49 \pm 3.8$ & $32 \pm 2.3$ & $42 \pm 6.2$ \\
\hline \multirow[t]{6}{*}{ TB10.4 } & IFN- $\gamma$ & $9,345 \pm 315$ & $1,058 \pm 35^{*}$ & $16,841 \pm 537^{*}$ & $16,856 \pm 414^{*}$ & $12,957 \pm 375^{*}$ \\
\hline & TNF- $\alpha$ & $2.62 \pm 0.18$ & $13.81 \pm 1.82^{*}$ & $2.14 \pm 0.13$ & $1.09 \pm 0.18$ & $12.40 \pm 0.20^{*}$ \\
\hline & IL-4 & $384 \pm 17$ & $30 \pm 0.7^{*}$ & $96 \pm 3.6^{*}$ & $63 \pm 3.4^{*}$ & $90 \pm 4.8^{*}$ \\
\hline & IL-5 & $5,111 \pm 46$ & $113 \pm 5.2^{*}$ & $5,071 \pm 203$ & $5,371 \pm 189$ & $3,799 \pm 21^{*}$ \\
\hline & IL-6 & $110 \pm 4.6$ & $134 \pm 17.4$ & $101 \pm 2.9$ & $134 \pm 5.7$ & $149 \pm 10.2$ \\
\hline & IL-10 & $100 \pm 2.5$ & $27 \pm 2.7^{*}$ & $33 \pm 1.2^{*}$ & $21 \pm 1.5^{*}$ & $27 \pm 3.5^{*}$ \\
\hline
\end{tabular}

Results represent the mean and SD of five animals per group. Asterisks indicate significant difference in cytokine secretion compared with the BCG-immunized group ( $p$ value $<0.05$, Student's $t$-test). Unit: $\mathrm{pg} / \mathrm{ml}$.

stimulation can slightly increase the secretion of IL-4 and IL-10 and significantly increase the secretion of IL-5 and IL-6 in the AdH4-MVAH4 group compared with that in the BCG group. However, TB10.4 significantly decreased the secretion of IL-4 and IL-10 in the viral vector-based vaccineimmunized groups relative to that in the BCG group. These data suggested that the characteristics of cytokine secretion in different immunized groups induced by Ag85B and TB10.4 stimulation are diverse. The Ad-containing vaccine regimens might induce IFN- $\gamma$-mediated immunity more easily than that by the MVAH4-MVAH4 vaccine regimen, and the AdH4-MVAH4 prime-boost regimen may induce a stronger Th1-mediated immune response than that by BCG.

\section{Discussion}

We developed two viral vector-based vaccines expressing an Ag85B-TB10.4 fusion protein, AdH4 and MVAH4, and investigated the impact of various prime-boost immunization regimens on the efficacy of these vaccines. We found that these vaccines can induce the production of significantly higher levels of antigen-specific antibodies, IFN- $\gamma$-producing splenocytes, and $\mathrm{CD} 69^{+} \mathrm{CD} 8^{+} \mathrm{T}$ cells, and IFN- $\gamma$ secretion when compared with that by the BCG-immunized mice. These results are in line with those reported by previous studies $[13,28]$.

In our previous study, we evaluated heterologous primeboost regimens by priming with BCG or recombinant BCG and boosting with recombinant MVA and/or Ad. We found that boosting mice with Ad85B-ESAT6 and MVA85BESAT6 vaccines can strengthen the BCG-induced immune protective effect [7]. In this study, we found that the AdH4containing vaccine immunization regimens (AdH4-AdH4, AdH4-MVAH4, and MVAH4-AdH4) induced significantly stronger antibody responses, higher production levels of IFN- $\gamma$-producing splenocytes and $\mathrm{CD} 69^{+} \mathrm{CD} 8^{+} \mathrm{T}$ cells, and higher levels of IFN- $\gamma$ secretion compared with MVAH4MVAH4 immunization. The results indicate that an immunization regimen containing AdH4 may have a higher capacity to induce mice humoral and cellular immune responses against TB than immunization regimens containing BCG or MVAH4 alone. Previous studies showed that repeated homologous immunization can reduce the breadth of immune responses, whereas a heterologous prime-boost regimen can generate a comprehensive immune response against Plasmodium falciparum TRAP [29]. Here, we found that levels of Th1-related cytokines (IFN- $\gamma$ and TNF- $\alpha$ ) in the AdH4-MVAH4- and MVAH4-MVAH4-immunized groups were significantly higher than those in the BCGimmunized groups. However, the AdH4-MVAH4-immunized mice showed significantly higher levels of IFN- $\gamma$ than MVAH4-MVAH4-immunized mice. Additionally, only IFN- $\gamma$ secretion was elevated significantly by AdH4-AdH4 and MVAH4-AdH4 immunization. The number of IFN- $\gamma$ producing splenocytes sensitive to $\mathrm{CD}^{+} \mathrm{T}$-cell restricted peptides of Ag85B (9-1p and 9-2p) in the AdH4-MVAH4immunized group was significantly higher than that in other viral vector-based vaccine-immunized groups. Previous 
studies report that the Th1-mediated immune response and $\mathrm{CD}^{+} \mathrm{T}$ lymphocytes are essential for the containment of M.tb infection [30]. These data imply that the heterologous prime-boost regimens using AdH4 for priming followed by boosting with MVAH4 may enhance the protective efficacy. However, one limitation of our study is that the immune protective effect of these vaccines and the various immunization regimens against M.tb infection have not been evaluated.

Several factors may affect the immune efficacy of $\mathrm{AdH} 4$ and MVAH4, such as the profile of antigen expression, the persistence of antigen, and the type of dendritic cells [31]. Previous studies have tried to explore the reason for the differences between immune responses elicited by Ad5- and MVA-based vaccines and they found some inconsistent results [31-33]. Maeda et al. [32] reported that the $\mathrm{Ad}$ vector elicits more antigen-specific IFN- $\gamma$ - producing T cells despite producing less antigen expression. However, Pillai et al. [31] found that the Ad5 vector can prime 6-fold higher levels of antigen-specific CD8 effector T cells than the MVA vector, but the difference of the antigen expression between Ad- and MVA-infected cells is not significant. Therefore, the mechanism for the immune response induced by these two viral-based vectors is complicated, and the detail and precise reason for why AdH4-containing immunization has a higher capacity to induce immune responses than MVAH4 alone needs to be further investigated.

In conclusion, both AdH4- and MVAH4-based vaccines can induce a humoral immune response against Ag85BTB10.4 in mice, and all these vaccines elicited a balanced Th1 and Th2 immune response. Moreover, the Ad-based vaccines might have a higher capacity to induce mouse cellular immune responses against TB than the BCG and MVAH4 vaccines.

\section{Acknowledgments}

This work was supported by the National Natural Science Foundation (Grant No. 81401717) of P. R. China.

\section{Conflict of Interest}

The authors have no financial conflicts of interest to declare.

\section{References}

1. WHO. Global tuberculosis report 2017. Available from http://www.who.int/tb/publications/global_report/en/.
Accessed March 09, 2018.

2. Brewer TF. 2000. Preventing tuberculosis with bacillus Calmette-Guerin vaccine: a meta-analysis of the literature. Clin. Infect. Dis. 31: S64-S67.

3. Xie Y, Chakravorty S, Armstrong D, Hall S, Via L, Song T, et al. 2017. Evaluation of a rapid molecular drug-susceptibility test for tuberculosis. N. Engl. J. Med. 377: 1043-1054.

4. Triccas JA, Counoupas C. 2016. Novel vaccination approaches to prevent tuberculosis in children. Pneumonia (Nathan) 8: 18.

5. Bo M, Zotti CM. 2016. European policies on tuberculosis prevention in healthcare workers: which role for BCG? A systematic review. Hum. Vaccin. Immunother. 12: 2753-2764.

6. Mendez-Samperio P. 2016. Global efforts in the development of vaccines for tuberculosis: requirements for improved vaccines against Mycobacterium tuberculosis. Scand. J. Immunol. 84: 204-210.

7. You Q, Wu Y, Wu Y, Wei W, Wang C, Jiang D, et al. 2012. Immunogenicity and protective efficacy of heterologous prime-boost regimens with mycobacterial vaccines and recombinant adenovirus- and poxvirus-vectored vaccines against murine tuberculosis. Int. J. Infect. Dis. 16: e816-e825.

8. Dean G, Clifford D, Gilbert S, McShane H, Hewinson RG, Vordermeier HM, et al. 2014. Effect of dose and route of immunisation on the immune response induced in cattle by heterologous Bacille Calmette-Guerin priming and recombinant adenoviral vector boosting. Vet. Immunol. Immunopathol. 158: 208-213.

9. Stylianou E, Griffiths KL, Poyntz HC, Harrington-Kandt R, Dicks MD, Stockdale L, et al. 2015. Improvement of BCG protective efficacy with a novel chimpanzee adenovirus and a modified vaccinia Ankara virus both expressing Ag85A. Vaccine 33: 6800-6808.

10. Minhinnick A, Satti I, Harris S, Wilkie M, Sheehan S, Stockdale L, et al. 2016. A first-in-human phase 1 trial to evaluate the safety and immunogenicity of the candidate tuberculosis vaccine MVA85A-IMX313, administered to BCG-vaccinated adults. Vaccine 34: 1412-1421.

11. Nemes E, Hesseling AC, Tameris M, Mauff K, Downing K, Mulenga $\mathrm{H}$, et al. 2018. Safety and immunogenicity of newborn MVA85A vaccination and selective, delayed bacille Calmette-Guerin for infants of human immunodeficiency virus-infected mothers: a phase 2 randomized, controlled trial. Clin. Infect. Dis. 66: 554-563.

12. Tye GJ, Lew MH, Choong YS, Lim TS, Sarmiento ME, Acosta A, et al. 2015. Vaccines for TB: lessons from the past translating into future potentials. Clin. Dev. Immunol. 2015: 916780.

13. Billeskov R, Christensen JP, Aagaard C, Andersen P, Dietrich J. 2013. Comparing adjuvanted H28 and modified vaccinia virus Ankara expressing $\mathrm{H} 28$ in a mouse and a non-human primate tuberculosis model. PLoS One 8: e72185.

14. Luabeya AK, Kagina BM, Tameris MD, Geldenhuys H, Hoff ST, Shi Z, et al. 2015. First-in-human trial of the post- 
exposure tuberculosis vaccine H56:IC31 in Mycobacterium tuberculosis infected and non-infected healthy adults. Vaccine 33: 4130-4140.

15. Li W, Deng G, Li M, Zeng J, Zhao L, Liu X, et al. 2014. A recombinant adenovirus expressing CFP10, ESAT6, Ag85A and Ag85B of Mycobacterium tuberculosis elicits strong antigen-specific immune responses in mice. Mol. Immunol. 62: 86-95.

16. Harth G, Lee BY, Wang J, Clemens DL, Horwitz MA. 1996. Novel insights into the genetics, biochemistry, and immunocytochemistry of the 30-kilodalton major extracellular protein of Mycobacterium tuberculosis. Infect. Immun. 64: 30383047.

17. Karbalaei Zadeh Babaki M, Soleimanpour S, Rezaee SA. 2017. Antigen 85 complex as a powerful Mycobacterium tuberculosis immunogene: biology, immune-pathogenicity, applications in diagnosis, and vaccine design. Microb. Pathog. 112: 20-29.

18. Lin PL, Dietrich J, Tan E, Abalos RM, Burgos J, Bigbee C, et al. 2012. The multistage vaccine $\mathrm{H} 56$ boosts the effects of BCG to protect cynomolgus macaques against active tuberculosis and reactivation of latent Mycobacterium tuberculosis infection. J. Clin. Invest. 122: 303-314.

19. Agger EM, Rosenkrands I, Olsen AW, Hatch G, Williams A, Kritsch C, et al. 2006. Protective immunity to tuberculosis with Ag85B-ESAT-6 in a synthetic cationic adjuvant system IC31. Vaccine 24: 5452-5460.

20. Ko A, Wui SR, Ryu JI, Lee YJ, Hien DTT, Rhee I, et al. 2018. Potentiation of Th1-type immune responses to Mycobacterium tuberculosis antigens in mice by cationic liposomes combined with de-O-acylated lipooligosaccharide. J. Microbiol. Biotechnol. 28: 136-144.

21. Dietrich J, Aagaard C, Leah R, Olsen AW, Stryhn A, Doherty TM, et al. 2005. Exchanging ESAT6 with TB10.4 in an Ag85B fusion molecule-based tuberculosis subunit vaccine: efficient protection and ESAT6-based sensitive monitoring of vaccine efficacy. J. Immunol. 174: 6332-6339.

22. Billeskov R, Elvang TT, Andersen PL, Dietrich J. 2012. The HyVac4 subunit vaccine efficiently boosts BCG-primed antimycobacterial protective immunity. PLoS One 7: e39909.

23. Skeiky YA, Dietrich J, Lasco TM, Stagliano K, Dheenadhayalan V, Goetz MA, et al. 2010. Non-clinical efficacy and safety of HyVac4:IC31 vaccine administered in a BCG prime-boost regimen. Vaccine 28: 1084-1093.

24. Geldenhuys H, Mearns H, Miles DJ, Tameris M, Hokey D, Shi Z, et al. 2015. The tuberculosis vaccine H4:IC31 is safe and induces a persistent polyfunctional CD4 T cell response in South African adults: a randomized controlled trial. Vaccine 33: 3592-3599.

25. Kou Y, Xu Y, Zhao Z, Liu J, Wu Y, You Q, et al. 2017. Tissue plasminogen activator (tPA) signal sequence enhances immunogenicity of MVA-based vaccine against tuberculosis. Immunol. Lett. 190: 51-57.

26. You Q, Jiang C, Wu Y, Yu X, Chen Y, Zhang X, et al. 2012. Subcutaneous administration of modified vaccinia virus Ankara expressing an Ag85B-ESAT6 fusion protein, but not an adenovirus-based vaccine, protects mice against intravenous challenge with Mycobacterium tuberculosis. Scand. J. Immunol. 75: 77-84.

27. Billeskov R, Grandal MV, Poulsen C, Christensen JP, Winther N, Vingsbo-Lundberg C, et al. 2010. Difference in TB10.4 T-cell epitope recognition following immunization with recombinant TB10.4, BCG or infection with Mycobacterium tuberculosis. Eur. J. Immunol. 40: 1342-1354.

28. Radosevic K, Wieland CW, Rodriguez A, Weverling GJ, Mintardjo R, Gillissen G, et al. 2007. Protective immune responses to a recombinant adenovirus type 35 tuberculosis vaccine in two mouse strains: CD4 and CD8 T-cell epitope mapping and role of gamma interferon. Infect. Immun. 75: 4105-4115.

29. Rollier CS, Hill AVS, Reyes-Sandoval A. 2016. Influence of adenovirus and MVA vaccines on the breadth and hierarchy of T cell responses. Vaccine 34: 4470-4474.

30. Zeng G, Zhang G, Chen X. 2018. Th1 cytokines, true functional signatures for protective immunity against $T B$ ? Cell. Mol. Immunol. 15: 206-215.

31. Pillai VK, Kannanganat S, Penaloza-Macmaster P, Chennareddi L, Robinson HL, Blackwell J, et al. 2011. Different patterns of expansion, contraction and memory differentiation of HIV-1 Gag-specific CD8 T cells elicited by adenovirus type 5 and modified vaccinia Ankara vaccines. Vaccine 29: 5399-5406.

32. Maeda K, West K, Hayasaka D, Ennis FA, Terajima M. 2005. Recombinant adenovirus vector vaccine induces stronger cytotoxic T-cell responses than recombinant vaccinia virus vector, plasmid DNA, or a combination of these. Viral Immunol. 18: 657-667.

33. Betts G, Poyntz H, Stylianou E, Reyes-Sandoval A, Cottingham M, Hill A, et al. 2012. Optimising immunogenicity with viral vectors: mixing MVA and HAdV-5 expressing the mycobacterial antigen Ag85A in a single injection. PLoS One 7: e50447. 